with oral acyclovir. Secondary bacterial infection occurred in $9 \%$ of the episodes. Disseminated disease or mortality was not reported in any patients. Significant post-herpetic neuralgia developed in $9 \%$ of the episodes. Patients with $\mathrm{HZ}$ reactivation were more likely to have first-time renal disease $(76 \%$ vs $58 \% ; \mathrm{p}=0.02)$ and a shorter SLE duration at $L N(31.4 \pm 50$ vs $62.7 \pm 72$ months; $p=0.02)$ than those without $H Z$. A trend of higher SLEDAI score, higher anti-dsDNA titer, lower C3 and albumin level but higher rate of refractory renal disease was also observed in $\mathrm{HZ}$-infected patients. Other clinical parameters such as histological classes of LN, neutrophil, lymphocyte counts and immunoglobulin levels at baseline and 6 months post-therapy were not significantly different between $\mathrm{HZ}$-infected and control patients. $\mathrm{HZ}$-infected patients had been treated with a significantly higher dose of prednisolone $(0.72 \pm 0.40$ vs $0.63 \pm 0.24 \mathrm{mg} /$ $\mathrm{kg} /$ day) as induction therapy. Dosages of other immunosuppressive drugs were not associated with $\mathrm{HZ}$ reactivation. Logistic regression revealed first-time renal disease (OR 2.25[1.08-4.71]; $p=0.003$ ), peak MMF daily dose (OR 1.24[1.10-3.07]; $p=0.02$ ) and cumulative CYC dose (OR 1.14[1.01-1.28]; $p=0.04$ ) during induction therapy were significantly associated with $\mathrm{HZ}$ within 2 years.

Conclusion: $\mathrm{HZ}$ reactivation is fairly common in $\mathrm{LN}$ patients undergoing immunosuppressive therapies but unpredictable from histological and laboratory parameters. Higher doses of prednisolone, MMF and CYC were associated with a higher risk of $\mathrm{HZ}$ reactivation within 2 years.

Acknowledgments: NIL

Disclosure of Interests: None declared

DOI: 10.1136/annrheumdis-2020-eular.2572

\section{THU0280 \\ IMPACT ON PHYSICIAN GLOBAL ASSESSMENT ON REMISSION RATES IN SLE. ANALYSIS FROM A GERMAN SLE-COHORT.}

J. Mucke ${ }^{1}$, C. Duesing ${ }^{1}$, M. Schneider ${ }^{1}$, G. Chehab ${ }^{1} .{ }^{1}$ Heinrich-Heine-University Duesseldorf, Policlinic and Hiller Research Unit for Rheumatology, Duesseldorf, Germany

Background: Defining remission for SLE as a suitable target for a treat to target (T2T) approach has been a major challenge in the past years. A few years back, four definitions of remission were presented by the international DORIS task force.[1] Parameters included in the definition are clinical activity (cSLE$\mathrm{DAI}$ ), steroid dose, immunosuppressive therapy, serology and physician global assessment (PGA). In particular the PGA, its threshold and general utility have been and still are discussed controversially.

Objectives: It was our aim to evaluate the added value of PGA in remission assessment.

Methods: In this monocentric cross-sectional study, patients with SLE according to the 1997 American College of Rheumatology (ACR) criteria were enrolled and assessed between September 2016 and December 2017. Two different definitions of remission were applied. The internationally accepted DORIS remission and a modified DORIS remission excluding PGA. Factors influencing PGA were assessed in the entire cohort. Regression analyses were used to assess differences between patients in DORIS and modified DORIS remission.

Results: A total of 233 patients were included ( $87.6 \%$ female). 98 patients $(41.9 \%)$ fulfilled any of the four DORIS remission definitions, while 154 patients $(66.1 \%)$ were in any modified remission in which PGA was excluded. In general, PGA rating was associated with disease activity (clinical SLEDAI; $p=<0.0001$ ), depression (Center for Epidemiological Studies Depression Scale; $p=0.049$ ), pain reported by the patient (numeric rating scale; $p=<0.0001)$ and hypocomplementemia $(p=<0.0001)$. Damage (SLICC damage index, SDI) did not influence PGA $(p=0.98)$. Both, DORIS and modified DORIS remission were associated with lower damage $(p=0.026 ; p=0.003)$, lower pain on NRS $(p=0.001 ; p=0.013)$, normal complement $(p=0.0005 ; p=0.005)$ and better illness perception ( $p=0.006 ; p=0.023$ ). Patients in modified DORIS remission had a tendency for more immunosuppressive therapy $(p=0.046)$.

Conclusion: Exclusion of PGA in remission assessment led to an increased number of patients in remission. Clinical parameters and factors associated with DORIS remission vs. modified DORIS remission were similar, hence the added value of PGA in our cohort regarding remission assessment is questionable. The use and especially the correct threshold of PGA for remission still has to be discussed. References:

[1] van Vollenhoven, Ronald; Voskuyl, Alexandre; Bertsias, George; Aranow, Cynthia; Aringer, Martin; Arnaud, Laurent et al. (2017): A framework for remission in SLE: consensus findings from a large international task force on definitions of remission in SLE (DORIS). In: Annals of the rheumatic diseases 76 (3), S. 554-561. DOI: 10.1136/annrheumdis-2016-209519.

Disclosure of Interests: Johanna Mucke: None declared, Christina Duesing: None declared, Matthias Schneider Grant/research support from: GSK, UCB, Abbvie, Consultant of: Abbvie, Alexion, Astra Zeneca, BMS, Boehringer Ingelheim, Gilead, Lilly, Sanofi, UCB, Speakers bureau: Abbvie, Astra Zeneca, BMS, Chugai, GSK, Lilly, Pfizer, Sanofi, Gamal Chehab Grant/research support from: Grant/research support from: GlaxoSmithKline and UCB Pharma for performing the LuLa-study.

DOI: 10.1136/annrheumdis-2020-eular.3860

\section{THU0281 EXPLORING THE GENETIC DIVERSITY OF STAPHYLOCOCCUS AUREUS IN PATIENTS AFFECTED BY SYSTEMIC LUPUS ERYTHEMATOSUS: ASSOCIATION WITH DISEASE-RELATED FEATURES AND ACTIVITY}

G. Olivieri ${ }^{1}$, F. Ceccarelli ${ }^{1}$, A. Lo Presti ${ }^{2}$, S. Angeletti ${ }^{3}$, C. Perricone ${ }^{4}$, G. Iaiani ${ }^{5}$, L. De Florio ${ }^{3}$, F. Antonelli ${ }^{3}$, L. Amori ${ }^{6}$, C. Garufi ${ }^{1}$, F. R. Spinelli ${ }^{1}$, C. Alessandri ${ }^{1}$ G. Valesini ${ }^{1}$, M. Cicozzi ${ }^{7}$, F. Conti ${ }^{1} .{ }^{1}$ Lupus Clinic, Rheumatology, Rome, Italy;

${ }^{2}$ Istituto Superiore di Sanità, Rome, Italy; ${ }^{3}$ Unit of Clinical Laboratory, Campus Bio-Medico, Rome, Italy; ${ }^{4}$ Rheumatology, Perugia, Italy; ${ }^{5}$ Malattie Infettive e Tropicali, Azienda Policlinico Umberto I, Rome, Italy; ${ }^{6}$ Sanità Pubblica e Malattie Infettive, Sapienza Università di Roma, Rome, Italy; ${ }^{7}$ Medical Statistics and Molecular Epidemiology, Campus Bio-Medico, Rome, Italy

Background: Infective factors play a central role in autoimmune diseases pathogenesis. It is possible to speculate that the host genotype could interact with genetic background of infective agents. We previously evaluated a large SLE cohort, observing the association between the S. Aureus (SA) carriage status and presence of a more active disease in terms of autoantibodies positivity.

Objectives: We evaluated epidemiological, molecular characterization, genetic diversity and evolution of SA isolated from SLE patients by means of phylogenetic analysis.

Methods: Consecutive SLE patients (ACR 1997 criteria) were enrolled: clinical/ laboratory data were collected and nasal swab for SA identification was performed. On the basis of translation elongation factor (tuf) gene, a phylogenetic analysis was performed to investigate phylogenetic relationships and to assess significant clades in patients with persistent carriage status (nasal swab positive in two consecutive evaluation, performed 1 week apart). The first dataset was composed by seven SA tuf gene isolated from non-SLE individuals from different countries (downloaded from the GenBank database, https://www.ncbi.nlm.nih.gov/nucleotide/) and tuf gene SA collected from SLE patients enrolled in the present study. Results: We enrolled 118 patients (M/F 10/198; median age 45.5 years, IQR 13,2; median disease duration 120 months, IQR 144). Skin involvement is the most frequent disease manifestation (86 patients, $72.9 \%$ ), followed by joint involvement (78 patients, $66.1 \%)$. Twenty-four patients $(20.3 \%$ ) were SA carriers $(\mathrm{SA}+)$, three of them resulted MRSA. SA+ patients showed a significantly higher prevalence of joint involvement $(79.2 \%$ versus $62.7 \%, \mathrm{P}=0.01)$ and anti-dsDNA positivity $(75.0 \%$ versus $55.3 \%, \mathrm{P}=0.004)$. Moreover, $\mathrm{SA}+\mathrm{SLE}$ showed a more active disease, in terms of SLEDAI-2k values [SA+: median 2 (IQR 3.75) versus SA-: median 0 (IQR 2), $\mathrm{P}=0.04$ ). The phylogenetic analysis has been restricted on the 21 non-MRSA SA+ patients. The maximum likelihood phylogenetic tree of the first dataset revealed a statistically supported larger clade $(A, N=17)$ and a smaller one $(B, N=4$; figure $1 A)$. SLE patients located in the clade $A$ showed a significantly higher prevalence of joint involvement $(88.2 \%)$ in comparison with clade $\mathrm{B}(50.0 \%, \mathrm{P}<0.0001)$ and $\mathrm{SA}-(62.7 \%, \mathrm{P}<0.0001$, figure $2 \mathrm{~B})$. Moreover, haematological manifestations were significantly more frequent in clade $A$ patients $(64.7 \%)$ compared with $\mathrm{B}(50.0 \%, \mathrm{P}=0.004$, figure $2 \mathrm{C})$.

Conclusion: The results of the present study confirmed the association between SA carriage status and disease activity, in terms of SLEDAI-2k values and antidsDNA positivity. The phylogenetic analysis on tuf gene show a clustering of $S A+$ patients in two major clade (A and $B$ ). Interestingly the tuf genotype of clade $A$ is significantly associated with a specific disease phenotype, characterized by joint involvement and positivity for anti-dsDNA. These findings support the hypothesis that bacterial genetic variants may be associated with specific disease features. References:

[1] Rigante et al. Int J Mol Sci. 2015;

[2] Wertheim et al Lancet Infect Dis. 2005;

[3] Conti et al Arthritis Res Ther 2016;

[4] Tong et al Clin Microbiol Rev. 2015;

[5] Rhee et al Infect Control Hosp Epidemiol. 2015
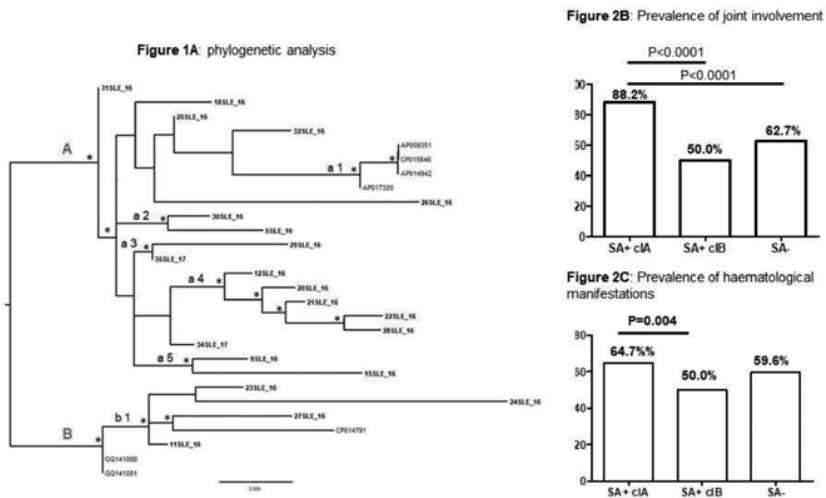

manifestation

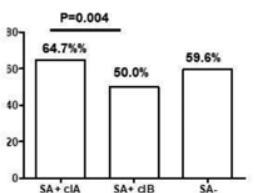

Legend $C A$, clade $A ; C B$, clade $B$ 